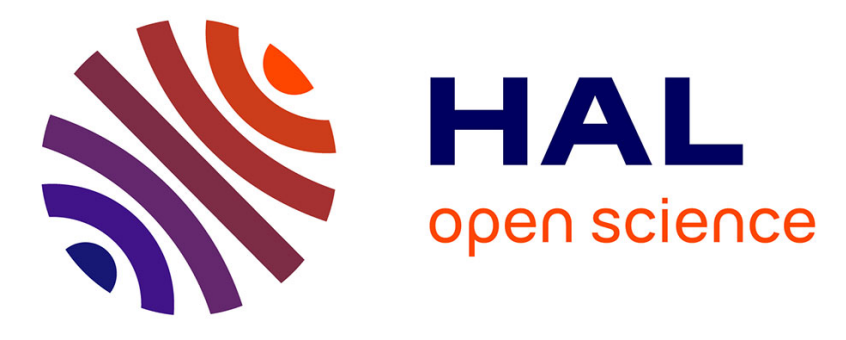

\title{
Decrypting the communication between microbes and the intestinal mucosa-A brief review on Pathogénie Microbienne Moléculaire's latest research
}

\author{
Aline Stedman, Katja Brunner, Giulia Nigro
}

\section{- To cite this version:}

Aline Stedman, Katja Brunner, Giulia Nigro. Decrypting the communication between microbes and the intestinal mucosa-A brief review on Pathogénie Microbienne Moléculaire's latest research. Cellular Microbiology, 2019, 21 (11), pp.e13118. 10.1111/cmi.13118 . pasteur-02548984

\section{HAL Id: pasteur-02548984}

\section{https://hal-pasteur.archives-ouvertes.fr/pasteur-02548984}

Submitted on 24 Apr 2020

HAL is a multi-disciplinary open access archive for the deposit and dissemination of scientific research documents, whether they are published or not. The documents may come from teaching and research institutions in France or abroad, or from public or private research centers.
L'archive ouverte pluridisciplinaire HAL, est destinée au dépôt et à la diffusion de documents scientifiques de niveau recherche, publiés ou non, émanant des établissements d'enseignement et de recherche français ou étrangers, des laboratoires publics ou privés. 


\title{
Decrypting the communication between microbes and the intestinal mucosa - A brief review on PMM's latest research
}

\author{
Aline Stedman ${ }^{1,2}$, Katja Brunner $^{1}$, and Giulia Nigro ${ }^{1}$ \\ ${ }^{1}$ Molecular Microbial Pathogenesis Unit, Institut Pasteur, INSERM U1202, 75015 \\ Paris, France \\ ${ }^{2}$ Current affiliation: Sorbonne Université, CNRS, Institut de Biologie Paris-Seine, \\ Paris, France
}

\begin{abstract}
Over the past 10 years, the "Pathogénie Microbienne Moléculaire" (PMM) unit of Prof. Philippe Sansonetti has studied the molecular cross-talk between the intestinal microbiota and the gut epithelium aiming to better understand how this mutualistic symbiosis delineates homeostasis and, when perturbed, prompts pathology. To do so, the unit has manipulated both bacterial and epithelial cells, and used cutting-edge technology. More recently, the lab has turned its focus also on studying the intestinal crypt and more specifically the intestinal stem cell (ISC), for their role in epithelial regeneration and long-term epithelium renewal. Here, we provide abrief review summarizing recent results obtained from the lab, with particular focus on the intestinal crypt.
\end{abstract}

\section{INTRODUCTION}

The microbiota is a complex ecosystem inhabiting a specific area of the body (e.g. skin, oral cavity, intestine) and is comprised of a diverse 
community of microorganisms, predominately bacteriabut also including others such as archaea, fungi, protozoa and viruses. The best studied to-date is the intestinal microbiota, in particular the one of the colon, where Bacteroidetes and Firmicutes represent the dominant phyla.

The microbiota has been referred to as the "forgotten organ" (O'Hara and Shanahan, 2006) as it plays a central role in health and disease by maintaining constant communication with the host epithelium either directly, or via bacterial products and metabolites.

Within the vast arsenal of microorganisms populating the gut, it is important to define categories exceeding species and genera-based definitions to encompass functionality and potential contribution to a healthy steadystate. The so-called commensals (e.g. Lactobacilli and Bifidobacteria) have established a symbiotic interaction with the host, while pathobionts, as is the case for the segmented filamentous bacteria (SFB), have the ability to promote immune maturation, but can also drive chronic inflammation, in the susceptible host. Pathogens, like Shigella flexneri on the other hand, are defined as microorganisms able to break the homeostatic barrier causing acute inflammation and infection. Among them, opportunistic pathogens take advantage of environmental changes, which may derive from a (transient) weakening of the host immune defense, an altered microbiota or physical damages, as in the example of Streptococcus gallolyticus (Hornef, 2015; Aymeric and Dramsi, 2018; Schnupf et al., 2018). The microbiota provides a natural defense barrier against pathogens by mechanisms, coining the term "colonization resistance", which include the occupation of common ecological niches and receptors, or the production of growth inhibitors, e.g. bacteriocins (Corr et al., 2007; Kommineni et al., 2015). In addition, it has important metabolic functions such as the fermentation of non-digestible dietary residues, vitamin production, and the detoxification of dietary carcinogens (Sansonetti, 2008). The microbiota influences specific physical properties of 
the intestinal barrier, such as the composition and thickness of the mucus layer, epithelial tight junctions (Ulluwishewa et al., 2011; Jakobsson et al., 2015) and it also interacts with the mucosal immune system by stimulating its development and maturation (Chow et al., 2010). Notably, recent studies report the multifaceted actions of the microbiome to extend the gut, by showing its impact on host behavior and global metabolism. Overall, the microbiota is a crucial component of a healthy intestinal environment (Tremaroli and Bäckhed, 2012; Sommer and Bäckhed, 2013). Changes in its composition complexity and/or distribution, leading to the loss of beneficial bacteria in advantage of pathobionts, or global microbiota impoverishment (i.e. dysbiosis) are correlated with a multitude of diseases, including diabetes, obesity, atherosclerosis, and also colon cancer (Karlsson et al., 2013; Vonaesch et al., 2018).

The gut microbiota is present all along the intestinal tract but differs notably depending on its location in the small intestine (duodenum, jejunum and ileum) or the large intestine (caecum, colon and rectum). Generally speaking, the microbiota increases in terms of complexity and concentration from the duodenum to the colon. Moreover, in each region there is a different local distribution of the microbiota due to morphological differences between small and large intestine. The small intestine is characterized by the presence of long epithelial protrusions extending into the intestinal lumen, the villi, which increase the total surface area of the epithelium and therefore enable maximal nutrient absorption, which is the primary function of this region. Both the small and large intestine contain epithelial invaginations along the mucosa, called intestinal crypts, which harbor proliferating progenitors and intestinal stem cells (ISC) at the bottom. ISCs are essential for the regeneration and repair of the intestinal tissue. Importantly, the ISC region of the small intestinal crypts also contains Paneth cells, which support ISC regenerative properties by secreting growth factors and metabolites, but also control the 
microbial environment of ISCs by the production of antimicrobial peptides (AMPs) (Sato et al., 2011; Clevers and Bevins, 2013).

The interaction between the microbiota and intestinal cells (immune and epithelial) is primarily maintained by host innate immune receptors (pattern recognition receptors - PRRs) that recognize conserved bacterial and viral motifs defined as "microbe-associated molecular patterns" (MAMPs) (Kawai and Akira, 2011). The PRR family is comprised of membrane bound receptors, notably Toll-like receptors (TLRs), and intracellular receptors such as nucleotide oligomerization domain receptors (NODs), cGAS or RIG-I-like receptors $(R L R)$, which are involved in the sensing of bacteria outside and inside the cells. MAMP recognition by PRRs canonically initiates an inflammatory signaling cascade via activation of the transcription factor NF$\kappa \mathrm{B}$ (nuclear factor-kappa $\mathrm{B}$ ) and subsequent production of pro-inflammatory cytokines, including IL-1 $\beta$, IL-6, or IFN- $\gamma$. However, in the context of gut health, PRRs have been associated with an additional function by promoting a continuous bacterial-host communication crucial for the maintenance of intestinal homeostasis (Abreu et al., 2005; Kawai and Akira, 2011).

Several studies have shown that bacteria or bacterial motifs play an essential role in host intestinal development, which includes the epithelial lining but also the immune system. While the recognition of the commensal microflora by epithelial TLRs is required for intestinal homeostasis (RakoffNahoum et al., 2004), the interaction between fragments of peptidoglycan and NOD1 receptors is essential and exclusive in inducing the genesis of isolated lymphoid follicles (Bouskra et al., 2008).

As previously mentioned, bacterial metabolites are important players in the microbiota-epithelium dialogue, which includes a multitude of small molecules such as bacteriocins, short chain fatty acids (SCFA), amino acids, or vitamins - some of which having an additional function for the host metabolism. For instance, in addition to serving as main energy substrate for 
colonocytes (Roediger, 1982), the SCFA butyrate, a potent histone deacetylase inhibitor, has important anti-inflammatory properties by inhibiting the expression of pro-inflammatory cytokines in macrophages (Chang et al., 2014) and by promoting the maturation of regulatory $\mathrm{T}$ cells (Treg) (Furusawa $e t$ al., 2013). Butyrate was also shown to induce the expression of the antimicrobial molecule cathelicidin LL-37 in colonocytes (Schauber et al., 2003).

More recently, butyrate was found to promote cell cycle arrest and differentiation of colonic progenitor cells (Kaiko et al., 2016), suggesting that it may also directly influence epithelial homeostasis. The concentration and diversity of bacterial metabolites is heavily dependent on the diet, promoting the notion that a dietary induced over-representation of specific bacterial metabolites might be involved in certain pathogeneses.

So-far, the extensive body of evidence confirming the microbialintestinal cross-talk has primarily been focusing on differentiated epithelial cells, i.e. enterocytes. Therefore it is important to also consider the impact on immature intestinal epithelial cells, including the stem cells residing in the intestinal crypts. It is interesting to hypothesize that stem cells may also respond to resident bacteria, either by direct recognition or via bacterial metabolites. If so, this is suggestive of a conserved host-bacterial interplay, which may have emerged from the coevolution of mammals with their gut microbiota. The involved regulatory systems must be tightly controlled and highlight a delicate balance by, on the one hand, protecting the vulnerable crypt stem cells from microbial insults while, on the other hand, enabling the sensing and integration of "positive" microbial signals to boost epithelial regeneration.

Here we review the recent work from our laboratory, "Pathogénie Microbienne Moléculaire" (PMM) unit headed by Philippe Sansonetti, focusing on the molecular cross-talk that characterize the mutualistic signals 
exchanged between commensals and the host. We describe novel tools, established by the laboratory, in order to functionally characterize commensal organisms such as Lactobacillus casei and SFB. We also report results on how specific symbionts affect the regulation of epithelial cell homeostasis and show vice-versa, how changes in host homeostasis alter the composition of the flora, possibly accelerating pathologic development. Finally, we delineate the identification and characterization of a specific murine intestinal crypt microbiota and present evidence indicating that an intimate dialogue between bacteria and the crypt might impact ISCs fate and survival (Figure 1).

\section{A bacterial close-up - investigations on symbiotic microbes}

Setting out on elucidating the molecular mechanisms underlying the delicate cross-talk between symbiotic microbes and intestinal mucosa, the PMM unit started off by investigating interactions from the microbial point of view. For instance, the lab established tools to functionally characterize specific symbionts. Using Lactobacillus casei as a symbiotic model microorganism, Licandro-Seraut et al. performed a genome-wide analysis seeking to identify the functional phenotype required to establish symbiotic colonization of the gut (Licandro-Seraut et al., 2014). Utilizing a transposonbased mutagenesis approach, the authors constructed a novel L. casei collection encompassing 1100 mutant strains, which were then screened for their ability to efficiently colonize the ligated rabbit ileal loop. Interestingly, among the 47 genes identified as essential for $L$. casei colonization, the authors found a high representation of genes involved in carbohydrate metabolism, indicating that colonization and host cell homeostasis shared a common gene pool. This library could serve a double purpose by on the one hand, 
providing a unique tool to investigate the molecular basis involved in the stable establishment of L. casei in the gut, while on the other hand permitting the identification bacterial effector mediated modulation of the host physiology, for example by investigating host transcriptional response towards different mutants.

Another example for the class of commensals is the anaerobic Candidatus arthromitus, also known as segmented filamentous bacterium (SFB). Within the thousands of bacterial species present in the intestine, SFB stands out due to its unique ability to stimulate post-natal maturation of the B-and T-cell compartments and in particular, the induction of the Th17 response in the small intestine. Given SFB's unique impact on the immune system, researchers have tried for decades to obtain in vitro cultures in order to investigate its physiologic properties and the underlying mechanisms on the host. In 2015, Schnupf et al. published for the first time, the successful culture of mouse SFB ex vivo utilizing a SFB-host cell co-culturing system in order to mimic its ecological niche (Schnupf et al., 2015). The authors were able to reproduce each of the SFB cell cycle steps, from the filaments to the differentiated, intracellular offspring. Moreover, they confirmed the potent stimulation of host innate defense genes by SFB, proving the system's suitability for further mechanistic studies on host-bacterial interactions. Of note, although SFB DNA has been isolated from multiple vertebrates, its presence in the human intestine was only recently confirmed by sequencing, SEM and FISH technology, thus indicating a potential role in the modulation of human immunity (Chen et al., 2018).

The ability to cultivate and functionally characterize symbionts is a major step in understanding the colonization of particular niches by individual species and their differential contribution to host homeostasis. However, many symbiotic bacteria of the gut remain uncultivable and therefore their potential role in health and disease is still unexplored. 


\section{Players in health and disease - the microbial-host interface}

Recent publications by our lab and others have emphasized on the close interconnectivity of bacterial metabolites and the host epithelium and thus highlighting the importance of this interdependency in the establishment of specific bacterial communities. For instance, Matsuki and collaborators showed that co-culture of intestinal cell lines with either Lactobacillus casei or Bifidobacterium breve altered the expression of key cell cycle regulators (Matsuki et al., 2013). The two SCFAs acetate and lactate, produced upon Lactobacilli and Bifidobacteria fermentation of complex sugars, were sufficient to mediate Cyclins D1 and E1 down-regulation and to induce cellcycle arrest. These results show that SCFAs have an additional role to the provision of fuel for colonocytes, by also influencing cell behavior. Another example on the regulation of host cell homeostasis by bacterial symbionts was reported in the recent publication by Tazi et al., demonstrating the L. paracasei and E. coli-mediated regulation of the host epithelial fatty acid metabolism, both in vivo and in vitro (Tazi et al., 2018). Mice colonized with either E. coli or L. paracasei showed a decrease in the expression of important lipogenic regulators within intestinal cells, including PPAR transcriptional targets, SREBP1 and CHREBP. This phenotype was correlated with gene expression changes in the liver and, in the case of E. coli, with reduced circulating chylomicrons. Interestingly, in vitro, both strains led to the decrease of enterocyte lipid secretion through two distinct mechanisms. While L. paracasei increased fat storage, E. coli was shown to upregulate lipid catabolism. The divergence in this mechanistic phenotype suggests species- or phylumspecificity, indicating that the relative abundance of the individual phyla and 
their spatial distribution in the crypt-lumen axis bears importance to the bacterial impact on host homeostasis. Importantly, colonization of mice with L. paracasei was protective from high-fat diet (HFD) induced hypercholesterolemia and attenuated intestinal response to HFD showing that this symbiont may be beneficial upon certain metabolic changes in the host (Amar et al., 2011; Öner et al., 2014).

HFD can induce obesity and associated metabolic complications and has been linked to changes in the microbiota composition (Ley et al., 2005). To gain a better insight into the mechanisms involved, Tomas and co-authors showed that a period of 30 days of HFD intake proved sufficient to significantly shift the commensal bacterial composition marked by an increase in the relative abundance of Proteobacteria or Verrucomicrobia, a decrease in Bacteroidetes, as well as the complete absence of SFB in the ileum (Tomas et al., 2016). In addition, severe changes to the intestinal defenses were observed upon short-time exposure to HFD, including the down-regulation of antimicrobial peptide (AMP) expression, narrowing of the mucosal barrier and subsequent colonization of the intervillous regions by bacteria. Interestingly, both phenotypes were mechanistically uncoupled as changes to the defense system were largely dependent on the sensing of fat by PPARgamma, while those to the microbial composition were linked to dietinduced modifications to the metabolic substrates available in the lumen. Taken together, these results confirm the importance of diet at the interface between the intestine and the microbiota. However, since the microbial composition appears modified prior to the onset of diseases (Tomas et al., 2016), it also raises the question of a possible causative role for dysbiosis in pathogenesis-associated with HFD.

Dysbiosis could be directly or indirectly responsible for disease onset by providing a suitable environment for the growth of pathobionts or opportunistic pathogens. Aymeric et al. reported an example for such 
interactions in the context of intestinal tumorigenesis conferring growth advantage to the opportunistic pathogen Streptococcus gallolyticus (Aymeric et $a l ., 2018)$. Using mice genetically prone to colorectal cancer (CRC), the authors showed that gut colonization by S. gallolyticus was higher in tumor-bearing mice than in healthy controls. This advantage was linked to the upregulation of a bacteriocin ("gallocin") found constitutively expressed by S. gallolyticus. The authors showed that in the presence of tumors, there is an enrichment of bile acid, which is required for the activation of gallocin from its inactive state, therefore increasing the competitive capacity of Streptococcus over other bacteria (i.e. Enterococci). Confirming previous observations, these results indicate that the adhesion of $S$. gallolyticus to tumoral cells promotes their proliferation rate, supporting the definition of S. gallolyticus as opportunistic pathogen.

Research from our lab and others demonstrates the ability of some bacteria to profoundly impact the intestinal metabolism and physiology. Correspondingly, it is tempting to speculate that intestinal crypts, having a central role in intestinal homeostasis, may also communicate with symbiotic bacteria and may therefore play an important part in the epithelial response towards the microbiota. Seeking to explore this hypothesis further, subsequent research activities by the laboratory aimed to investigate the existence and identity of bacteria in the vicinity of the crypt compartment.

\section{Bacteria in the Crypt - microbiota and intestinal regeneration}

The intestinal crypt represents a sensitive zone in the gut as it is home to the ISCs. Given its crucial importance for epithelial health, the lab decided to focus on this region and investigate a possible direct impact of the microbiota on intestinal regeneration.

In order to address the presence of bacteria within the intestinal crypts of mice, Pedron et al. applied a novel technical approach combining WhartinStarry (silver/nitrate) staining and fluorescent in situ hybridization (FISH) 
with 16SrRNA probes (Pédron et al., 2012). For the first time, the authors identified the presence of a small cluster of bacteria in the crypts and demonstrated their viability and metabolic activity. Interestingly, local differences were noted as bacteria were found at the crypt bottom in the caecum and proximal part of the colon, but absent in the duodenum-jejunum and distal colon. To identify the crypt bacteria, a dedicated pipeline was developed combining laser capture microdissection (LCM) and 16S rRNA metagenomics. The analysis was carried-out on mice from different genetic backgrounds and obtained from several providers to account for interspecies and environmental variations. Comparing samples obtained from luminal and crypt regions revealed the presence of fourteen bacterial phyla with predominance of five phyla: Firmicutes (73\%), Beta- and Gammaproteobacteria (16\%), Actinobacteria (3.5\%), and Bacteroidetes (1.7\%). Interestingly, marked differences were observed when comparing samples from the luminal and the crypt region of the same mouse as Firmicutes comprised the majority of sequences (95.5\%) in the former, while Proteobacteria represented the most abundant sequences (47.6\%, versus $2.7 \%$ for the lumen) in the latter region. Bacteroidetes were relatively poorly represented within both crypt and luminal samples. In the crypt, the most prominent bacterial family of Proteobacteria was the Moraxellaceae (23.7\%); of these, $23 \%$ identified as Acinetobacter spp. sequences in crypts versus $1.6 \%$ in the lumen. Shared Acinetobacter OTUs were found among all crypts suggesting a common bacterial phylogroup although quantitative variations between mice of different background were noted. In all cases, the levels of Acinetobacter spp. were significantly higher in crypt than in luminal samples. Interestingly, Acinetobacter spp. belong to the family of strictly aerobic, nonfermenting gamma-proteobacteria suggesting an oxygen enriched environment in the proximity of the crypt.

In order to investigate the potential benefits of these specific crypt 
microbes on the host, Saffarian et al. characterized and compared the genome of two different strains of Acinetobacter (A. modestus CM11G and A.

radioresistens $\mathrm{CM} 38.2$ ) that were isolated from murine crypts (Saffarian et al., 2017). Interestingly, both strains contained genes encoding for the presence of a type IV pili system as well as the presence of genes associated with Type I and Type II secretion systems. In contrast to these findings, pathogenic Acinetobacter bacteria, including A. baumannii, possess a T6SS associated to bacterial virulence (Repizo et al., 2015). Most importantly, the authors highlighted the potential of crypt Acinetobacter to metabolize xenobiotics due to the expression of the benABCDE operon (involved in benzoate metabolism), antABC operon (encoding the anthranilate dioxygenase) and, in the case of $A$. radioresistens, the phenol hydroxylase gene cluster. Comparative genomic analysis revealed that different Acinetobacter strains isolated from the same biological niche, even though sharing the large majority of genes, possess unique features that may play a specific role in the protection of the intestinal crypt.

Altogether, these results suggest that among the complex assemblage of luminal and mucosal commensal species composing the microbiota, a limited set of individual species may enter into a mutualistic interaction with their host, potentially reflecting a longstanding co-evolution. This may have led to the established symbiotic steady-state in which the epithelial regenerative apparatus may benefit from microbiota-mediated protection and vice-versa. These findings illustrate the importance of further investigations in order to establish a cartography of commensals permitting a better understanding on symbiosis.

In order to decipher the significance of the CSCM-crypt symbiosis, we explored the functional characterization of this community, i.e. the existence of a true cross/talk between the microbiota and the cells of the crypt. For this, Naito et al. have selected four members of each of the three main genera 
identified in the crypts: Acinetobacter, Stenotrophomonas, and Delftia and investigated their impact on the colonic epithelium in vivo and in vitro (Naito et al., 2017). Given the implication of bacterial products (especially the endotoxin) and the TLRs on intestinal homeostasis, the authors utilized germfree mice and monocolonized them with A. modestus, A. radioresistens, $D$. tsuruhatensis, and S. maltophilia followed by FISH analysis. Bacteria were detected in the luminal space of the intestine, but also deep in the colonic crypts, thus confirming the crypt tropism of these four species. In alignment with previous results, crypts of the small intestine (duodenum, jejunum, and ileum) and distal colon remained devoid of bacteria following monocolonization. Analyzing the impact of crypt colonization on colon epithelial turnover, Naito et al. observed that CSCM members led to a decrease in the number of proliferating cells and an increase in the number of dead cells. Interestingly, these observations were found to be TLR4-dependent, in vivo. Subsequently, LPS from the selected CSCM strains were purified and evaluated for their effect on colonic organoid culture. In agreement with the colonization model, treating organoids with the purified LPS, composed of highly acylated lipid A, had a cytotoxic effect on ISCs and, most strikingly, on progenitor cells through a mechanism identified as necroptosis. In addition to the decrease of proliferative cells, cell differentiation was observed to be increased and most profoundly towards the goblet cell lineage. These observations imply that the intimate relationship between crypts and a subset of colonic microbes could be important to regulate the turnover rate of ISCs at homeostasis but may also affect cell differentiation. How the CSCM impacts on ISCs self-renewal in a context of active epithelial regeneration remains an important question to address.

In addition to LPS, other MAMPs produced by the CSCM may have a direct impact on ISCs. To investigate this question, Nigro et al. subjected murine small intestinal organoids to several bacterial products and assessed 
their effects on organoid survival (Nigro et al., 2014). In contrast to the cell toxicity observed by LPS, treatment with muramyl-dipeptide (MDP) component of peptidoglycan resulted in higher yields of organoids, indicating a protective role for MDP on ISCs. The authors showed that this mechanism was NOD2-dependent and further confirmed the NOD2 gene to be highest expressed in ISCs when compared to Paneth cells. Using co-culture of sorted ISCs and Paneth cells from wt and/or NOD2KO mice, Nigro et al. showed that direct sensing of MDP by NOD2 expressing ISCs conferred the protective phenotype. Notably, this MDP-mediated protection was shown to be important upon stress, i.e. following the isolation protocol, but not in steadystate condition, as indicated by the normal appearance and viability of NOD2KO mice. To validate this NOD2-dependent ISCs cytoprotection pathway and to assess its activation by microbiota-produced MDP in vivo, the authors utilized a NOD2KO mouse model and applied the chemotherapeutic agent doxorubicin, to induce oxidative stress in proliferative cells.

Interestingly, in comparison to wt mice, NOD2KO mice lacked the ability to regenerate the gut upon treatment with doxorubicin. Moreover, the wt mice presented higher numbers of crypt survival compared to NOD2KO mice, indicating a protective effect of NOD2. Crypts extracted from doxorubicintreated mice were much more responsive to MDP-dependent organoid proliferation when compared to non-treatment further confirming the notion of stress-dependence of the NOD2-cytoprotective pathway. These direct interactions between bacterial products and ISCs highlight the potential of the microbiota to impact ISCs development and therefore to affect mucosal health (Nigro et al., 2014). The molecular mechanisms underlying this cytoprotective pathway in ISCs are subject of current investigations in the lab (Levy et al, under review). A closer contact of microbiota with the intestinal crypt has been observed in several conditions including HFD or adenomas formation (Ley et al., 2005). Therefore, at the onset of pathogenesis the interactions 
between certain bacterial MAMPs and the ISCs may be altered when compared to homeostasis, with potential to greatly impact the physiological outcome. While ISCs express a number of PRRs, the expression level of specific receptors vary depending on their location in the anteroposterior axis of the intestine. In addition, our results suggest that PRR activation in ISCs might trigger alternative pathway activation as compared to other cell types in the intestine. Therefore, there is a need for future research to further define the scope of these interactions and to elucidate the molecular implications on the activation processes within ISCs.

\section{CONCLUSIONS}

Recent work by our unit, has set the intestinal crypt in the center of the microbiota-epithelial dialogue. In addition to having an essential role in epithelial regeneration, crypt stem cells were shown to possess the ability to sense bacteria and bacterial signaling, potentially affecting epithelial physiology and importantly, long-term memory. To investigate these associations further, it is now crucial to develop novel, more integrated analysis tools allowing for the ex vivo study of this system. For instance, one approach could lie in the adaptation of the "gut on a chip" technology, which is currently being developed by our laboratory. Another point of focus should be the identification and functional analysis of the most important symbionts required for host homeostasis, with particular emphasis on the CSCM. Finally, a better understanding of the mechanisms underlying signaling distribution throughout the three players (microbes, immune and epithelial cells) and how they integrate at the level of the crypt will be essential to explain the onset and impact of dysbiosis. Joint efforts and a multidisciplinary approach will be needed to then extend these concepts to other mammalian species, including humans. 


\section{Acknowledgments}

This work was supported by French National Research Agency (ANR) grant 17-CE14-0022 (i-Stress).

\section{Conflict of Interest}

The authors declare no conflict of interest. 
FIGURE LEGEND

Figure 1. Schematic summary of the recent publications by the PMM lab depicted in the relevant location along the intestine. 


\section{REFERENCES}

Abreu, M.T., Fukata, M., and Arditi, M. (2005) TLR signaling in the gut in health and disease. J Immunol 174: 4453-4460.

Amar, J., Chabo, C., Waget, A., Klopp, P., Vachoux, C., Bermúdez-Humarán, L.G., et al. (2011) Intestinal mucosal adherence and translocation of commensal bacteria at the early onset of type 2 diabetes: molecular mechanisms and probiotic treatment. EMBO Mol Med 3: 559-572.

Aymeric, L., and Dramsi, S. (2018) Streptococcus gallolyticus: an opportunistic pathogen associated with colorectal cancer. Med Sci (Paris) 34: 784-787.

Aymeric, L., Donnadieu, F., Mulet, C., Merle, du, L., Nigro, G., Saffarian, A., et al. (2018) Colorectal cancer specific conditions promote Streptococcus gallolyticus gut colonization. Proc Natl Acad Sci 115: E283-E291.

Bouskra, D., Brézillon, C., Bérard, M., Werts, C., Varona, R., Boneca, I.G., and Eberl, G. (2008) Lymphoid tissue genesis induced by commensals through NOD1 regulates intestinal homeostasis. Nature 456: 507-510.

Chang, P.V., Hao, L., Offermanns, S., and Medzhitov, R. (2014) The microbial metabolite butyrate regulates intestinal macrophage function via histone deacetylase inhibition. Proc Natl Acad Sci 111: 2247-2252.

Chen, B., Chen, H., Shu, X., Yin, Y., Li, J., Qin, J., et al. (2018) Presence of Segmented Filamentous Bacteria in Human Children and Its Potential Role in the Modulation of Human Gut Immunity. Front Microbiol 9: 1403.

Chow, J., Lee, S.M., Shen, Y., Khosravi, A., and Mazmanian, S.K. (2010) Hostbacterial symbiosis in health and disease. Adv Immunol 107: 243-274.

Clevers, H.C., and Bevins, C.L. (2013) Paneth cells: maestros of the small intestinal crypts. Annu Rev Physiol 75: 289-311.

Corr, S.C., Li, Y., Riedel, C.U., O'Toole, P.W., Hill, C., and Gahan, C.G.M. (2007) Bacteriocin production as a mechanism for the antiinfective activity of Lactobacillus salivarius UCC118. Proc Natl Acad Sci USA 104: 7617-7621.

Furusawa, Y., Obata, Y., Fukuda, S., Endo, T.A., Nakato, G., Takahashi, D., et al. (2013) Commensal microbe-derived butyrate induces the differentiation of colonic regulatory T cells. Nature 504: 446-450. 
Hornef, M. (2015) Pathogens, Commensal Symbionts, and Pathobionts: Discovery and Functional Effects on the Host. ILAR J 56: 159-162.

Jakobsson, H.E., Rodríguez-Piñeiro, A.M., Schütte, A., Ermund, A., Boysen, P., Bemark, M., et al. (2015) The composition of the gut microbiota shapes the colon mucus barrier. EMBO Rep 16: 164.

Kaiko, G.E., Ryu, S.H., Koues, O.I., Collins, P.L., Solnica-Krezel, L., Pearce, E.J., et al. (2016) The Colonic crypt protects stem cells from microbiota-derived metabolites. Cell 165: 1708-1720.

Karlsson, F., Tremaroli, V., Nielsen, J., and Bäckhed, F. (2013) Assessing the Human Gut Microbiota in Metabolic Diseases. Diabetes 62: 3341-3349.

Kawai, T., and Akira, S. (2011) Toll-like receptors and their crosstalk with other innate receptors in infection and immunity. Immunity 34: 637-650.

Kommineni, S., Bretl, D.J., Lam, V., Chakraborty, R., Hayward, M., Simpson, P., et al. (2015) Bacteriocin production augments niche competition by enterococci in the mammalian gastrointestinal tract. Nature 526: 719-722.

Ley, R.E., Bäckhed, F., Turnbaugh, P., Lozupone, C.A., Knight, R.D., and Gordon, J.I. (2005) Obesity alters gut microbial ecology. Proc Natl Acad Sci USA 102: 11070-11075.

Licandro-Seraut, H., Scornec, H., Pedron, T., Cavin, J.F., and Sansonetti, P.J. (2014) Functional genomics of Lactobacillus casei establishment in the gut. Proc Natl Acad Sci 111: E3101-E3109.

Matsuki, T., Pédron, T., Regnault, B., Mulet, C., Hara, T., and Sansonetti, P.J. (2013) Epithelial cell proliferation arrest induced by lactate and acetate from Lactobacillus casei and Bifidobacterium breve. PLoS ONE 8: e63053.

Naito, T., Mulet, C., De Castro, C., Molinaro, A., Saffarian, A., Nigro, G., et al. (2017) Lipopolysaccharide from Crypt-Specific Core Microbiota modulates the colonic epithelial proliferation-to-differentiation balance. mBio 8 .

Nigro, G., Rossi, R., Commere, P.-H., Jay, P., and Sansonetti, P.J. (2014) The cytosolic bacterial peptidoglycan sensor Nod2 affords stem cell protection and links microbes to gut epithelial regeneration. Cell Host Microbe 371: 792798.

O'Hara, A.M., and Shanahan, F. (2006) The gut flora as a forgotten organ. EMBO Rep 7: 688-693.

Tazi, A., Araujo, J.R., Mulet, C., Arena, E.T., Nigro, G., Pédron, T., and 
lowering effects of lactobacilli and bifidobacteria strains as potential probiotics with their bsh gene analysis. J Mol Microbiol Biotechnol 24: 12-18.

Pédron, T., Mulet, C., Dauga, C., Frangeul, L., Chervaux, C., Grompone, G., and Sansonetti, P.J. (2012) A Crypt-Specific Core Microbiota Resides in the Mouse Colon. mBio 3: e00116-12.

Rakoff-Nahoum, S., Rakoff-Nahoum, S., Paglino, J., Paglino, J., EslamiVarzaneh, F., Eslami-Varzaneh, F., et al. (2004) Recognition of commensal microflora by toll-like receptors is required for intestinal homeostasis. Cell 118: 229-241.

Repizo, G.D., Gagné, S., Foucault-Grunenwald, M.-L., Borges, V., Charpentier, X., Limansky, A.S., et al. (2015) Differential role of the T6SS in Acinetobacter baumannii virulence. PLoS ONE 10: e0138265.

Roediger, W.E. (1982) Utilization of nutrients by isolated epithelial cells of the rat colon. Gastroenterology 83: 424-429.

Saffarian, A., Touchon, M., Mulet, C., Tournebize, R., Passet, V., Brisse, S., et al. (2017) Comparative genomic analysis of Acinetobacter strains isolated frommurine colonic crypts. 1-12.

Sansonetti, P.J. (2008) Host-bacteria homeostasis in the healthy and inflamed gut. Current Opinion in Gastroenterology 24: 435-439.

Sato, T., van Es, J.H., Snippert, H.J., Stange, D.E., Vries, R.G., van den Born, M., et al. (2011) Paneth cells constitute the niche for Lgr5 stem cells in intestinal crypts. Nature 469: 415-418.

Schauber, J., Svanholm, C., Termén, S., Iffland, K., Menzel, T., Scheppach, W., et al. (2003) Expression of the cathelicidin LL-37 is modulated by short chain fatty acids in colonocytes: relevance of signalling pathways. Gut 52: 735-741.

Schnupf, P., Gaboriau-Routhiau, V., and Cerf-Bensussan, N. (2018) Modulation of the gut microbiota to improve innate resistance. Current Opinion in Immunology 54: 137-144.

Schnupf, P., Gaboriau-Routhiau, V., Gros, M., Friedman, R., Moya-Nilges, M., Nigro, G., et al. (2015) Growth and host interaction of mouse segmented filamentous bacteria in vitro. Nature 520: 99-103.

Sommer, F., and Bäckhed, F. (2013) The gut microbiota - masters of host development and physiology. Nat Rev Microbiol 11: 227-238.

Oner, O., Aslim, B., and Aydaş, S.B. (2014) Mechanisms of cholesterol- 
Sansonetti, P.J. (2018) Disentangling Host-Microbiota Regulation of Lipid Secretion by Enterocytes: Insights from Commensals Lactobacillus paracasei and Escherichia coli.mBio 9.

Tomas, J., Mulet, C., Saffarian, A., Cavin, J.-B., Ducroc, R., Regnault, B., et al. (2016) High-fat diet modifies the PPAR- $\gamma$ pathway leading to disruption of microbial and physiological ecosystem in murine small intestine. Proc Natl Acad Sci 113: E5934-E5943.

Tremaroli, V., and Bäckhed, F. (2012) Functional interactions between the gut microbiota and host metabolism. Nature 489: 242-249.

Ulluwishewa, D., Anderson, R.C., McNabb, W.C., Moughan, P.J., Wells, J.M., and Roy, N.C. (2011) Regulation of tight junction permeability by intestinal bacteria and dietary components. J Nutr 141: 769-776.

Vonaesch, P., Anderson, M., and Sansonetti, P.J. (2018) Pathogens, microbiome and the host: emergence of the ecological Koch's postulates. FEMS Microbiol Rev 42: 273-292.

Tazi, A., Araujo, J.R., Mulet, C., Arena, E.T., Nigro, G., Pédron, T., and 
Schematic summary of the recent publications by the PMM lab depicted in the relevant location along the intestine.

Aymeric et al., 2018

Colorectal cancer specific conditions promote Streptococcus gallolyticus gut colonization

Saffarian et al., 2017

Comparative genomic analysis of Acinetobacter strains isolated from murine colonic crypts

Matsuki et al., 2013

Epithelial cell proliferation arrest induced by lactate and acetate from Lactobacillus casei and Bifidobacterium breve

High-fat diet modifies the PPAR- $\gamma$ pathway

leading to disruption of microbial and

physiological ecosystem in murine small intestine

Nigro et al., 2014

The cytosolic bacterial peptidoglycan sensor NOD2 affords stem cell protection and links microbes to gut epithelial regeneration

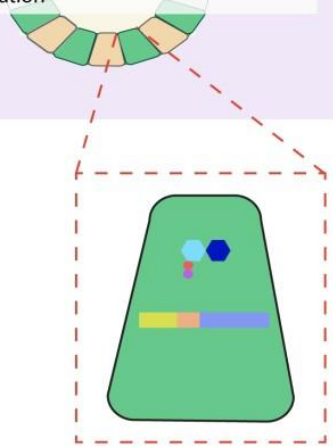

$\begin{array}{lll}\text { Intestinal stem cell } & \text { Small intestinal microbiota } \\ \text { POD2 } & \text { SFB }\end{array}$


56

57

59

60 